A Granular Intrusion Detection System using Rough Cognitive Networks Peer-reviewed author version

NAPOLES RUIZ, Gonzalo; Grau, Isel; Falcon, Rafael; Bello, Rafael \& VANHOOF, Koen (2016) A Granular Intrusion Detection System using Rough Cognitive Networks. In: Abielmona, Rami; Falcon, Rafael; Zincir-Heywood, Nur; Abbass, Hussein A. (Ed.). Recent Advances in Computational Intelligence in Defence and Security, p. 169-191.

DOI: 10.1007/978-3-319-26450-9_7

Handle: http://hdl.handle.net/1942/21458 


\section{ResearchGate}

See discussions, stats, and author profiles for this publication at:

https://www.researchgate.net/publication/283505893

\section{A Granular Intrusion Detection System Using Rough Cognitive Networks}

Chapter · January 2016

5 authors, including:

\section{Rafael Falcon}

Larus Technologies Corpora...

69 PUBLICATIONS 246 CITATIONS

SEE PROFILE

Koen Vanhoof

Hasselt University

243 PUBLICATIONS 1,998

CITATIONS

SEE PROFILE 


\title{
A Granular Intrusion Detection System Using Rough Cognitive Networks
}

\author{
Gonzalo Nápoles, Isel Grau, Rafael Falcon, Rafael Bello \\ and Koen Vanhoof
}

G. Nápoles $\cdot$ I. Grau $\cdot$ R. Bello

Computer Science Department, Central University of Las Villas, Carretera

Camajuaní Km 5.5, 54830 Santa Clara, Cuba

e-mail: gnapoles@uclv.edu.cu

I. Grau

e-mail: igrau@uclv.edu.cu

R. Bello

e-mail: rbellop@uclv.edu.cu

R. Falcon (汭)

Research \& Engineering Division, Larus Technologies Corporation, 170 Laurier Ave. West - Suite 310, Ottawa, ON K1P 5V5, Canada e-mail: rafael.falcon@larus.com; rfalcon@uottawa.ca

\section{R. Falcon}

Electrical Engineering \& Computer Science, University of Ottawa, 800 King Edward Ave., Ottawa, ON K1N 6N5, Canada

\section{G. Nápoles · K. Vanhoof}

Hasselt Universiteit Campus Diepenbeek, Agoralaan Gebouw D,

BE3590 Diepenbeek, Belgium

e-mail: gonzalo.napoles@ student.uhasselt.be

K. Vanhoof

e-mail: koen.vanhoof@uhasselt.be

(C) Springer International Publishing Switzerland 2016

R. Abielmona et al. (eds.), Recent Advances in Computational Intelligence

in Defense and Security, Studies in Computational Intelligence 621,

DOI 10.1007/978-3-319-26450-9_7 
as the collected data is still permeated with uncertainty. In this chapter, we tackle the network intrusion detection problem from a classification angle by using a recently proposed granular model named Rough Cognitive Networks (RCN). An RCN is a fuzzy cognitive map that leans upon rough set theory to define its topological constructs. An optimization-based learning mechanism for RCNs is also introduced. The empirical evidence indicates that the RCN is a suitable approach for detecting abnormal traffic patterns in computer networks.

Keywords Intrusion detection system - Computational intelligence - Granular computing $\cdot$ Rough set theory $\cdot$ Fuzzy cognitive maps $•$ Rough cognitive networks Harmony search

\section{Introduction}

The 21 st century has brought forth a digital age in which we are all immersed. Up-and-coming information communication and processing paradigms such as the Internet of Things (IoT) [4], Cloud Computing [47], Software-Defined Networks [32] and Wearable Computing [25] are increasingly gaining momentum and rapidly permeating every facet of mankind. These new architectural frameworks bring a unique set of challenges with them, among which cybersecurity is one of paramount importance. The computer systems that constitute the backbone of critical infrastructure behind a plethora of industrial and societal processes often become prey to sophisticated malicious attacks that originate at any node in the entangled World Wide Web. As a result, governments and businesses are adapting their legislative bodies to account for the prevention, detection and mitigation of the risks and threats associated with these potentially devastating attacks [39].

Intrusion Detection Systems (IDS) [43] have become an essential component of security infrastructure to detect these threats before they inflict widespread damage, since traditional approaches (e.g., access control, encryption, firewalls, etc.) are unable to completely protect networks from attacks and malwares. The purpose of an IDS is to analyze the network traffic, either the incoming one or existing logs of past traffic activities, and identify anomalous behaviours that could reasonably be taken as cues of the presence of an intruder in the system. Concisely described, network intrusion detection is essentially a pattern recognition problem in which network traffic patterns are classified as either normal or abnormal.

Although traditional statistical techniques have enjoyed success in analyzing traffic flows as part of an IDS operation, the network security research community is increasingly leaning on Computational Intelligence (CI) solutions due to their ability to adapt to complex environments, handle noise and uncertainty and remain computationally tractable and robust.

More recently, the advent of Granular Computing (GrC) $[6,26,52]$ as an innovative information representation and processing framework has largely influenced the way CI systems are being conceived nowadays. This is due to the fact that 
GrC provides reasoning constructs at higher levels of abstraction that better capture human understanding of the real world. From classification [55] to clustering [51], time-series prediction [72] and decision making [50], granular models are becoming prominent tools for the analysis of large volumes of data as they operate upon information granules (i.e., constructs of order higher than plain numeric or symbolic atoms) and can better represent and manifest the dynamics of human-centric world modeling.

In this chapter, we tackle network intrusion detection via a $\mathrm{GrC}$ model and demonstrate its advantages over several traditional classification schemes. Our study makes the following contributions: (1) we model network intrusion detection as a classification problem and apply a recently introduced granular model, named "Rough Cognitive Network"(RCN), to the analysis of archived traffic data in computer networks for intrusion detection purposes; (2) we put forth a learning mechanism for RCNs that is based on self-adaptive Harmony Search [44]; (3) we empirically evaluate the RCN performance in conjunction with that of seven well-established classifiers in the literature. The experimental evidence confirms that RCNs are a plausible model to discriminate between normal and abnormal traffic patterns in network data as it attains high detection rates (i.e., successfully identified abnormalities) and low false negative rates (misidentified anomalies).

The rest of this chapter is structured as follows. Section 2 briefly surveys relevant works in intrusion detection systems, with special emphasis on CI-based solutions. Section 3 elaborates on the two precursor formalisms leading up to RCNs: rough set theory (RST) and fuzzy cognitive maps (FCMs). Then, the RCN topology learning and classification inference process are dissected in Sect. 4 while Sect. 5 describes the proposed optimization-based RCN parameter learning method. The experimental analysis is unveiled in Sect. 6 before conclusions and future work directions are outlined in Sect. 7.

\section{Related Work}

In this section, we briefly review several published works that are relevant to our study. They provide the necessary background to understand the contents of this chapter.

\subsection{Intrusion Detection Systems}

The literature in the IDS arena is quite vast. This field appears often interwoven with other similar terms such as "network anomaly detection" or "network intrusion detection" and the common underlying problem has been addressed through a myriad of techniques. In a recent and comprehensive survey [8] covering publications in this field from 2000 to 2012, $28 \%$ of the papers surveyed approached IDS from 
a supervised learning angle (i.e., classification), as we do in this chapter. However, unsupervised learning (via clustering) was the preferred choice of $21 \%$ of the papers given that labeled data could be scarce and/or difficult to access in certain cases where privacy concerns impede the sharing of such information.

The statistical methods and systems applied to intrusion detection $[45,61,66$, 79] first construct a general statistic model of the observed traffic data, either via parametric techniques (which assume the knowledge of the type of probability distribution is available and then try to learn their parameters) or by means of nonparametric techniques, which do not lay any assumption on the type of the data distribution. Once this model has been fitted to the data, any point (traffic pattern) with low probability of having been generated by the underlying data model is labeled as an outlier and hence flagged as suspicious.

The use of computational intelligence methods in the IDS realm has been well documented in the 2010 survey compiled by Wu and Banzhaf [73]. Artificial neural networks (ANNs) [11, 40, 67, 78, 81], fuzzy sets [16, 21, 29, 68], evolutionary computation [5, 18, 24, 31, 38, 57-59], artificial immune systems (AIS) [70, 75], fuzzy cognitive maps [62-64, 74, 83], rough sets [2, 13, 14] and swarm intelligence (SI) $[19,20,29]$ techniques, all representative methods of the wider CI/Soft Computing (SC) family, and their hybrids [15, 22, 63, 64, 74] have all been wielded against complex network traffic datasets to identify attack vectors or suspicious activities either in a supervised or unsupervised fashion.

\subsection{Rough Set Theory in Network Security}

Rough sets and fuzzy cognitive maps have been independently applied to network intrusion detection $[8,73]$, although the number of reported works thus far is not significant compared to the volume of documented applications of other CI techniques.

Chen et al. [13] employ rough set theory in the preprocessing stage of their proposed network intrusion detection scheme in order to remove irrelevant attributes prior to the operation of the Support Vector Machine (SVM)-based classifier. A similar use (attribute dimensionality reduction) is evoked by Li and Zhao with their Fuzzy SVM [41] and by Zhang et al. in the context of their Artificial Immune System (AIS)-based technique [82], where the number of attributes that describe an antibody is shortened using the lower and upper approximations of each rough concept. Shrivastava and Jain [60] also boost the network traffic classification power of their SVM via rough-set-based feature selection by dropping 35 irrelevant attributes out of 41 initially gathered to describe the traffic flows in their system. An analogous rationale is pursued by Sivaranjanadevi et al. in their work [65] and by Poongothai and Duraiswamy in [53]. 
Fuzzy and rough sets are integrated into a partitive clustering engine in [14] to address network intrusion detection from an unsupervised perspective; the proposed clustering method yielded superior results compared to other classical unsupervised techniques.

Finally, rough sets are used in [2] to induce classification rules via the LEM2 algorithm so as to create a potent classifier capable of detecting network intrusions with high detection rate and low false alarm rate. The classification results of LEM2 are found to be more interpretable and can be obtained in a shorter time than those of the K-nearest neighbor classifier, which are more accurate yet more resourcedemanding.

\subsection{Fuzzy Cognitive Maps in Network Security}

Xin et al. [74] derive fuzzy features from the network data and pass them on to a fuzzy cognitive map (FCM) in order to model more complex attack vectors.

Siraj et al. [63] used FCM and fuzzy rule bases to model causal knowledge among different intrusion variables in an interpretable fashion. Suspicious events are mapped to nodes in FCM, which function as neurons that trigger alerts with different weights depicting on the causal relations between them. So, an alert value for a particular machine or a user is calculated as a function of all the activated suspicious events at a given time. This value reflects the safety level of that machine or user at that time.

Siraj et al. [64] chose FCMs and fuzzy rule bases as the vehicles for causal knowledge acquisition within the decision engine of an intelligent IDS deployed at the Mississipi State University. The system fuses information from a variety of intrusion detection sensors. In particular, the FCMs are used at two levels: (i) to model individual suspicious events such as 'high login failure' or 'SYN flood' and (ii) to ascertain the overall impact of various suspicious events (input concepts) for each host computer and system user (output concepts).

Afterwards, Siraj and Vaughn [62] also leaned upon FCMs to cluster network intrusion alerts based on discovered similarities among the raw features extracted from sensor data. The FCM is thus acting as a fusion machine where intrusion evidence for a particular network resource that originates at different clusters is amalgamated.

Zhong et al. [83] consider a distributed attack scenario and resort to an FCM to describe the entities that are part of it as well as their relationships.

The study authored by Jazzar and Bin Jantan [27] focuses on IDS designed around the Self Organizing Map (SOM) neural network given its ability to process large volumes of data with low computational overhead. Having realized that these systems still exhibit a high false alarm rate, they coupled the SOM with an FCM in order to refine the clustering performed by the former approached. The FCM's role is to calculate the relevance of odd concepts (neurons) to a network attack. By doing so, 
irrelevant concepts can be left out and other concepts may come to the forefront of the intrusion analysis.

Krichene and Boudriga [37] devised a methodology to automatically determine responses to security incidents. The underlying formalism that allows attack identification, complexity reduction and response elicitation is termed an incident response probabilistic cognitive map. These maps differ from traditional FCMs in that they are capable of modeling different relationships between symptoms, actions and unauthorized results as pertaining to a network attack. A function that enables the identification of those concepts that are tied to a set of events is also part of the proposed scheme. The authors illustrate their proposal on a real-world denial of service (DoS) attack against a web server.

Zaghdoud and Al-Kahtani [80] bring forth a multi-layered architecture for intrusion detection and response. They employ an FCM to gauge the impact of a confirmed intrusion event belonging to a known class upon the compromised system. The FCM nodes represent components of the computer network system or security concepts whereas the edges symbolize the influence exercised by one component upon another; these influences must be carefully taken into consideration now that a network intrusion has been confirmed.

\subsection{Discussion}

Our proposed granular classifier, the Rough Cognitive Network, borrows from both aforementioned techniques: RST and FCM; however, their synergy is dictated by a topological arrangement of the FCM nodes into symbolic and higher-order information granules, the latter of which correspond to the three RST-based regions (positive, boundary, negative) of the decision concepts (classes) induced by a similarity relationship over the set of input attributes in the data set under consideration. To the best of our knowledge, this hybridization scheme is completely different from previous efforts to combine both methodologies, and so is certainly the RCN application to the IDS domain.

\section{The Forerunners of Rough Cognitive Networks}

As mentioned before, in this paper we design an IDS which uses an RCN for detecting potentially atypical (and likely dangerous) patterns. One could briefly define an RCN as a Sigmoid Fuzzy Cognitive Map where concepts represent granules of information. In this section, we summarize the mathematical underpinnings behind Rough Set Theory and Fuzzy Cognitive Maps, which are the two core building blocks of the granular model proposed in this chapter. 


\subsection{Rough Set Theory}

Rough Set Theory (RST) is a robust and mature theory for handling uncertainty in the form of inconsistency in the data [1, 49]. The RST framework employs two exact set approximations to describe a generic or real-world concept. Let us assume a decision system $S=(U, A \cup d)$, where $U$ is a non-empty finite set of objects called the universe, $A$ is a non-empty finite set of attributes, while $d \notin A$ denotes the decision attribute. Any subset $X \subseteq U$ can be approximated by two crisp sets: the lower and upper approximations. These sets are defined as $B_{*} X=x \in U:[x]_{B} \subseteq X$ and $B^{*} X=x \in U:[x]_{B} \cap X \neq \emptyset$ where the equivalence class $[x]_{B}$ comprises the set of inseparable objects associated to the target instance $x$ that are described using $B \subseteq A$.

Based on the lower and upper approximations, we can compute the positive, negative and boundary regions of any concept $X$. The positive region $\operatorname{POS}(X)=$ $B_{*} X$ includes those objects that are certainly contained in $X$; the negative region $N E G(X)=U-B^{*} X$ involves those objects that are certainly not contained in $X$, whereas the boundary region $B N D(X)=B^{*} X-B_{*} X$ represents the objects whose membership to the set $X$ is uncertain, i.e., they might be members of $X$. These regions are in fact information granules and provide a valuable knowledge when facing decision-making or pattern classification problems.

Based on the positive, negative and boundary regions, Yao [76] defined two types of rules: deterministic decision rules for the positive region and undeterministic decision rules for the boundary region. More recently Yao [77] introduced the three-way decisions model. Rules constructed from the three regions are associated with different actions [23]. A positive rule suggests a decision of acceptance, a negative rule makes a decision of rejection and a boundary rule implies a decision of abstaining. The three-way decisions play an important role in decision-making problems [42].

In the classical RST formulation, the indiscernibility relation is defined as an equivalence relation; hence, two objects will be inseparable if they are identical with respect to a set of attributes $B \subseteq A$. The equivalence relation $R$ induces a partition of the universe $U$ on the basis of the attributes in $B$. However, this definition is extremely strict. For example, a decision system with millions of objects will be categorized as inconsistent if two objects are equivalent but they have different decision classes (i.e., two experts might have different perceptions about the same observation). But are two objects really significant in a universe comprised of millions of objects?

To counter the above stringent definition, the equivalence requirement on $R$ is relaxed. In fact, if we adopt a "weaker" inseparability relation then we could tackle problems having numerical (or mixed) attributes. Two inseparable objects, according to some similarity relationship $R$, will be tossed together in the same set of not identical (but reasonably similar) instances. Equation 1 shows the indiscernibility relation adopted in this paper, where $0 \leq \varphi(x, y) \leq 1$ is a similarity function. This binary relation determines whether two objects $x$ and $y$ are inseparable or not (i.e., as long as their similarity degree $\varphi(x, y)$ is greater than or equal to a user-specified threshold $\xi$ ). 
Despite the clear advantages of using this approach to cope with problems having numerical features, selecting the correct value for the similarity threshold $\xi$ could be a challenge.

$$
R: y R x \Leftrightarrow \varphi(x, y) \geq \xi
$$

If the threshold $\xi=1$ then the similarity relation $R$ will be reflexive, transitive and symmetric, leading to Pawlak's model for discrete (nominal) domains. If $\xi<1$ then the similarity relation will be reflexive and symmetric but not transitive.

Another aspect to be considered when designing a similarity relation is the adequate selection of the similarity function. Equation 2 shows a variant which combines both numerical and categorical attributes. It provides a more general formulation for addressing decision-making problems having different features.

$$
\varphi(x, y)=\frac{1}{|A|} \sum_{i=1}^{|A|} \omega_{i} \delta(x(i), y(i)) .
$$

In the above equation, $A$ is the set of features describing the problem, $0 \leq \omega_{i} \leq 1$ represents the relative importance of the $i$ th attribute, $x(i)$ and $y(i)$ denote the values of the $i$ th attribute associated with the objects $x$ and $y$ respectively, and $\delta$ is the attribute-wise similarity function. The greater $0 \leq \varphi(x, y) \leq 1$, the more similar the objects $x$ and $y$. Equations 3 and 4 display the attribute-wise similarity functions adopted in this research study. The function $\delta_{1}$ is used when we want to compare two values of a discrete attribute, whereas $\delta_{2}$ is used for comparing two values of a numerical attribute $\left(L_{i}\right.$ and $H_{i}$ denote the lowest and highest value of the $i$ th attribute, respectively).

$$
\delta_{1}(x(i), y(i))=\left\{\begin{array}{l}
1, x(i)=y(i) \\
0, x(i) \neq y(i)
\end{array} .\right.
$$

$$
\delta_{2}(x(i), y(i))=1-\frac{|x(i)-y(i)|}{H_{i}-L_{i}} .
$$

Equations 5 and 6 respectively formalize how to compute the lower and upper approximations of a concept $X$, where $R(x)$ denotes the similarity class of the object $x$. These exact sets are the basis for granulating the available information about the concept using RST, and they become the core of Granular Fuzzy Cognitive Maps [48].

$$
\begin{gathered}
B_{*} X=\{x \in U: R(x) \subseteq X\} . \\
B^{*} X=\bigcup_{x \in X} R(x) .
\end{gathered}
$$

As a result, an object can simultaneously belong to multiple similarity classes, so the covering induced by the similarity relation $R$ over the universe $U$ is not necessarily a partition [7]. Therefore, similarity relations do not induce a partition of 
the universe, but rather generate similarity classes. It suggests that an object could simultaneously belong to different similarity classes, and consequently the instance $x$ could activate several granular regions. In such cases, the decision-making stage becomes really difficult for the expert, since it has to consider non-trivial decision patterns.

\subsection{Fuzzy Cognitive Maps}

Fuzzy Cognitive Maps (FCM) are recurrent neural networks for modeling and simulation [34] consisting of concepts and their causal relations. Concepts are equivalent to neurons denoting objects, variables, or entities related to the system under investigation whereas the weights associated with the connections among neurons denote the strength of the causality among such nodes. It should be highlighted that causal relations are quantified in the range $[-1 ; 1]$. This value is the result of the numerical evaluation of a fuzzy linguistic variable, which is usually assigned by experts during the modeling phase [36]. The activation value of the neurons is also fuzzy in nature and regularly takes values in the range $[0 ; 1]$ although the interval $[-1 ; 1]$ is used too. The magnitude of the activation is also meaningful for the model: the higher the activation value of a map concept, the stronger its influence over the system under consideration.

Equation 7 mathematically formalizes the rule for updating the activation value of concepts in an FCM, assuming $A^{0}$ is the initial configuration. This rule is iteratively repeated until a fixed point attractor or a maximum number of iterations $T$ is reached. At each step $t$ a new state vector is produced, and after a large enough number of iterations, the map will arrive at one of the following states: (i) fixed equilibrium point, (ii) limited cycle or (iii) chaotic behavior [35]. If the FCM reaches a fixedpoint attractor, then we can conclude that the map has converged. In such cases, the final output corresponds to the desired state (i.e., the system response for the activation vector).

$$
A_{i}^{t+1}=f\left(\sum_{j=1}^{M} w_{j i} A_{j}^{t}+w_{i i} A_{i}^{t}\right), i \neq j .
$$

In the above equation $f($.$) represents a monotonically non-decreasing nonlinear$ function which is used for transforming the activation value of each concept (the weighted combination of the activation levels). The most used functions are: the bivalent function, the trivalent function, and sigmoid variants [10]. In this paper we will focus on sigmoid functions since it has been shown that they exhibit superior prediction capabilities [10]. 


\section{Rough Cognitive Networks}

Rough Cognitive Networks (RCNs) [48] are an extension of three-way decision rules introduced by Yao [76]. In a nutshell, we can define an RCN as a sigmoid FCM where concepts denote information granules, namely, the RST-derived positive, boundary and negative regions of the original problem as well as the set of decision classes in the problem at hand.

The RCN methodology not just allows solving mixed-attribute problems, but also provides accurate inferences since it uses a recurrent inferential process to converge to a stable attractor, which comprises the most fitting decision class. It should be pointed out that the complexity of this model does not depend on the number of attributes in the decision system, but on the number of decision classes. In this section, we explain how to learn an RCN from data. Furthermore, we introduce a supervised learning algorithm for computing the required RCN parameters, which enhances the value of our proposal.

\subsection{Information Granulation and Network Design}

As mentioned before, a central aspect when designing an $\mathrm{RCN}$ is the process related to the construction of positive, negative and boundary regions. Let us assume a pattern classification problem and a partition $X=X_{1}, \ldots, X_{k}, \ldots, X_{N}$ of the universe $U$ according to the decision attribute, where each subset $X_{k}$ denotes a decision class and comprises all instances labeled as $d_{k}$. These information granules will be expressed as map concepts. More precisely, input concepts denote positive, negative and boundary regions associated with each subset $X_{k}$; they are subsequently used for activating the network.

In the RCN model, the output neurons do not influence other neurons since they are target concepts. Once the FCM inference process is done (this point will be clarified next), the activation degree of each output concept (decision class) will be gauged. After the map concepts are defined, we establish causal connections among such neurons, where the direction and intensity of the causal weights are computed according to the set of rules below:

- $R_{1}$ : IF $C_{i}$ is $P_{k}$ AND $C_{j}$ is $d_{k}$ THEN $w_{i j}=1.0$.

- $R_{2}$ : IF $C_{i}$ is $P_{k}$ AND $C_{j}$ is $d_{(v \neq k)}$ THEN $w_{i j}=-1.0$.

- $R_{3}$ : IF $C_{i}$ is $P_{k}$ AND $C_{j}$ is $P_{(v \neq k)}$ THEN $w_{i j}=-1.0$.

- $R_{4}$ : IF $C_{i}$ is $N_{k}$ AND $C_{j}$ is $d_{k}$ THEN $w_{i j}=-1.0$.

In the above rules, $C_{i}$ and $C_{j}$ denote two map concepts, $P_{k}$ and $N_{k}$ are the positive and negative region for the $k$ th decision respectively, whereas $-1 \leq w_{i j} \leq 1$ is the 
causal weight between the cause $C_{i}$ and the effect $C_{j}$. More precisely, rules $R_{1}$ and $R_{2}$ define the relation between positive regions and decision neurons. If the positive region $P_{k}$ is activated (rule 1), then the decision $d_{k}$ must be stimulated as well, since we confidently know that objects belonging to the positive region $P_{k}$ will be categorically members of the concept $X_{k}$. Accordingly, decisions $d_{(v \neq k)}$ must be inhibited (rule 2) because an object cannot simultaneously belong to different positive regions.

The third rule follows an analogous reasoning: if a positive region $P_{k}$ is activated then positive regions unrelated to the decision $d_{k}$ (i.e., $P_{(v \neq k)}$ ) will be inhibited. If the negative region $N_{k}$ is activated (rule 4 ), then the map will inhibit the decision, but we cannot conclude anything about other decisions. Moreover, we incorporated an additional rule for handling the intrinsic knowledge concerning the RST boundary regions:

- $R_{5}$ : IF $C_{i}$ is $B_{k} A N D C_{j}$ is $d_{v} A N D\left(B N D\left(X_{k}\right) \cap B N D\left(X_{v}\right) \neq \emptyset\right) T H E N w_{i j}=0.5$.

Observe that not all boundary regions are included in the RCN's topology. This is dictated by the learning procedure on the training data: if a boundary region is empty $\left(B N D\left(X_{k}\right)=\emptyset\right)$ then the neuron $B_{k}$ will be removed from the modeling in order to simplify the network topology. On the other hand, we need to establish causal links between each boundary neuron and decision classes involving some degree of uncertainty; otherwise the causal connection will be removed from the map as well.

The above topology construction scheme implies that an RCN for a problem with $|D|$ decision classes will have at most $3|D|$ input neurons (assuming all boundary regions are in), $|D|$ decision (output) neurons and $3|D|(1+|D|)$ causal relations. Additionally, for each neuron we add a self-reinforcement connection with causality $w_{i i}=1$ which partially preserves the initial excitation.

\subsection{Inference Using Rough Cognitive Networks}

The final phase concerns the network exploitation, where the activation value of input and decision concepts play a pivotal role. In this scheme, to classify a test instance $O_{i}$, first the excitation vector $A_{i}$ will be calculated using the similarity class $R\left(O_{i}\right)$ and its relation to each RST-based region. For instance, let us assume that $\left|P O S\left(X_{1}\right)\right|=20,\left|R\left(O_{i}\right)\right|=10$, whereas the number of objects that belong to the positive region is given by the expression: $\left|R\left(O_{i}\right) \cap \operatorname{POS}\left(X_{k}\right)\right|=7$. This implies that the activation degree of the neuron $P_{1}$ is $7 / 20=0.35$. It denotes the conditional probability of accepting $d_{1}$ given the similarity class $R\left(O_{i}\right)$, that is $\operatorname{Pr}\left(d_{k} \mid R\left(O_{i}\right)\right)$. Analogously, we can compute the activation degree of other input concepts related to each decision class. Rules $R_{6}-R_{8}$ formalize this procedure as follows:

- $R_{6}$ : IF $C_{i}$ is $P_{k}$ THEN $A_{i}^{0}=\frac{\left|R\left(O_{i}\right) \cap P O S\left(X_{k}\right)\right|}{\left|\operatorname{POS}\left(X_{k}\right)\right|}$.

- $R_{7}$ : IF $C_{i}$ is $N_{k}$ THEN $A_{i}^{0}=\frac{\left|R\left(O_{i}\right) \cap N E G\left(X_{k}\right)\right|}{\left|N E G\left(X_{k}\right)\right|}$.

- $R_{8}: I F C_{i}$ is $B_{k}$ THEN $A_{i}^{0}=\frac{\left|R\left(O_{i}\right) \cap B N D\left(X_{k}\right)\right|}{\left|B N D\left(X_{k}\right)\right|}$. 
Once the activation vector $A^{0}$ has been computed, we trigger the FCM inference rule until a fixed point attractor, or a maximal number of iterations $T$ is reached. This process will stress a pattern using the similarity class of the instance $O_{i}$ to do that, which is desirable in problems with insufficient positive evidence where selecting the proper class could be difficult. Afterward one can use the output vector for making a decision (e.g., we can sort the alternatives according to the preference degrees calculated by the map inference process). When dealing with pattern classification problems, the final output will be the concept having the highest activation, or alternatively it could be a random class if the input similarity class only activates negative and/or boundary regions.

\section{Learning Methodology for Rough Cognitive Networks}

As mentioned before, the basis for computing the set of positive, negative and boundary regions is the proper estimation of the similarity threshold $\xi$ in Eq. 1. If this value is too small then positive regions will be small as well, leading to poor excitation of neurons. This step is quite important when selecting the most adequate decision: the higher the activation of the positive region, the more desirable the decision (although the model will compute the final decision taking into account all the evidence). If this threshold $\xi$ is excessively large then boundary regions will be large, thus increasing the uncertainty.

In this section, we present a learning algorithm for tuning the model parameters, which is based on the Harmony Search (HS) metaheuristic [44]. The method needs to adjust two kinds of parameters: the weight $\omega_{i}$ of each attribute and the similarity threshold $\xi$. This approach leads to a numerical optimization problem with $|A|+1$ variables and will be solved using an adaptive variant of the HS procedure.

The HS metaheuristic is a simple-trajectory search method, which only evaluates one potential solution at a time, instead of evaluating a set of potential solutions (as it occurs with population-based metaheuristics). This HS design choice is relevant for our learning methodology since evaluating a solution means computing the set of lower and upper approximations, which could be computationally expensive as the number of objects in the training data set increase.

During the optimization phase, the algorithm randomly creates a harmony memory with size HMS and iteratively improves a new harmony from the HM. If the improved harmony is better than the worst harmony in the HM, then the new solution replaces the worst harmony. Despite its algorithmic simplicity, HS suffers from a serious problem common to other metaheuristics: its search capabilities are quite sensitive to the specified parameter vector.

For this reason in this paper we adopt an improved variant, called Self-adaptive Harmony Search (SHS), which is capable of adjusting its own parameters [71]. The SHS method not only alleviates the parametric sensitivity issue, but also significantly enhances the accuracy of the solutions. Algorithm 1 shows the pseudocode of this metaheuristic, where $N$ is the maximal number of iterations, HMCR 
428

429

(Harmony Memory Consideration Rate) is a parameter that controls the balance between exploitations and exploration, while $R_{1}=U-x$ and $R_{2}=x-L$, assuming that $L$ and $U$ respectively denote the lowest and the highest values for each problem variable in the harmony memory.

On the other hand, PAR is the pitch adjustment rate and determines whether further adjustment is required to a harmony drawn from the harmony memory. In this variant, the PAR factor is linearly decreased over time. Experiments reported by the authors [71] suggested that moderate size of the harmony memory (e.g., 50) and large values of HMCR (e.g., 0.9) are adequate choices for these parameters. Based on these considerations, we used these values during the experiments and simulations performed in the next section. The $\operatorname{rand}()$ function draws a random number uniformly distributed in the unit interval.

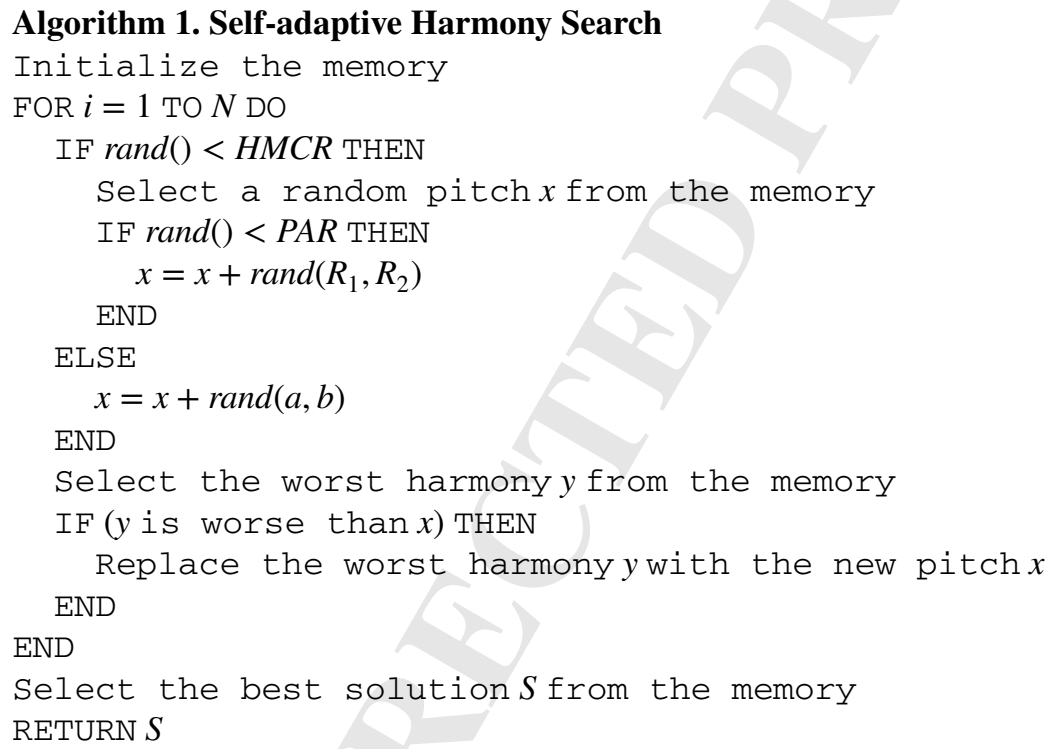

The other component of the optimization problem to be specified is the objective function. Equation 8 shows the function $G($.) used in this study, where the parameters denote the set of weights $W$, the similarity threshold $\xi$ and the set of instances $\phi$ to be used for training the model, respectively. On the other hand, $\aleph_{R(W, \xi)}(x)$ is the output vector computed by the RCN which is obtained from the similarity threshold defined by the function $R(W, \xi)$, whereas the function $Y(x)$ is the known class vector associated with the instance $x$ and $D$ is the set of decision classes in the problem. It should be also mentioned that $\|.\|_{L}$ refers to a norm (e.g., the $L_{1}$-norm, $L_{2}$-norm or $L_{\infty}$-norm) that is used to calculate the error.

$$
\operatorname{minimize} G(W, \xi, \phi)=\sum_{x \in \phi} \frac{\left\|\aleph_{R(W, \xi)}(x)-Y(x)\right\|_{L}}{|\phi||D|} \text {. }
$$


If $G(W, \xi, \phi)=0$ then the RCN, using the similarity relation $R$, is capable of recognizing all patterns stored in the training set; otherwise the value $1-G(W, \xi, \phi)$ stands for the model accuracy. The proposed parameter tuning method not only estimates the introduced parameters, but also allows determining the relevance of each attribute, which contributes to elicit further knowledge about the problem.

\section{Detecting Intrusion in Computer Networks}

In this section we study the performance of the proposed granular cognitive network for detecting abnormal traffic behavior in computer networks. As mentioned before, this problem can be envisioned as a challenging pattern classification task having two decision classes: either 'normal' or 'abnormal'. In order to perform our simulations, we used an improved variant of the NSL-KDD dataset [17] which is a widely used benchmark when testing IDS [19, 22, 23]. In the following section, we summarize the most important features of both training and testing NSL-KDD datasets.

\subsection{Description of the NSL-KDD Dataset}

Perhaps the most popular dataset for evaluating the performance of anomaly detection models is KDD'99 [30]. The KDD training dataset consists of 4,900,000 network connection vectors, each of which contains 41 features. Such features could be gathered in three groups: (i) basic features, (ii) traffic features and (iii) content features.

The first group comprises attributes extracted from a TCP/IP connection, whereas the second one includes time-based features computed in a window interval (e.g., connections in the past $2 \mathrm{~s}$ having the same destination host or the same service as the current connection). It should be stated that there are several slow-probing attacks that scan the ports using a much larger time interval than $2 \mathrm{~s}$ and accordingly these attacks will not produce any intrusion patterns. Finally, the third group contains features related to attacks having a single connection, which do not have intrusion frequent sequential patterns. In such cases, attacks are embedded in the data portions of packets, hence forcing the Intrusion Detection System to catch suspicious behavior in the data portion (e.g., number of failed login attempts) instead of in the connections.

On the other hand, in the training set each record is labeled as either "normal" or "abnormal" with exactly one specific attack type (i.e., Probing Attack, Denial of Service Attack, User to Root Attack and Remote to Local Attack).

It is essential to mention that the KDD'99 dataset was built based on the data captured in DARPA'98 which has been criticized by McHugh [46]. It suggests that some of the existing problems in the dataset DARPA'98 remain in KDD'99. More recently, Tavallaee and collaborators [69] conducted a statistical analysis where two 
important issues were detected. The first important deficiency in the KDD'99 dataset is the huge number of redundant records (78 and $75 \%$ of records are duplicated in the train and test set, respectively). Consequently, this will cause learning algorithms to be biased towards the more frequent records. As a second issue they noticed that this dataset has poor difficulty level: about $98 \%$ of the records in the train set and $86 \%$ of the records in the test set were correctly classified with 21 learned machines (7 learners, each trained 3 times with different training sets).

To solve the aforementioned issues, Tavallaee et al. [69] removed all the redundant records in both train and test sets. Moreover, they randomly sampled correctly classified records in such a way that the number of selected instances from each difficulty level group is inversely proportional to the percentage of records in the original dataset. This refinement process gave rise to two improved datasets called KDDTrain+ and KDDTest+ which include 125,973 and 22,544 records, respectively. As well, they created another test set called KDDTest- 21 by removing the records that were correctly classified by all 21 learners. This dataset contains 11,850 records, which are more difficult to classify. Because of its increasing popularity and sound verification procedure, we adopted Tavallaee et al's data sets for our experimentation.

\subsection{Numerical Simulations}

Next we study the behavior of RCN across the selected dataset. Figure 1 displays the network topology that allows solving the prediction problem (i.e., where each

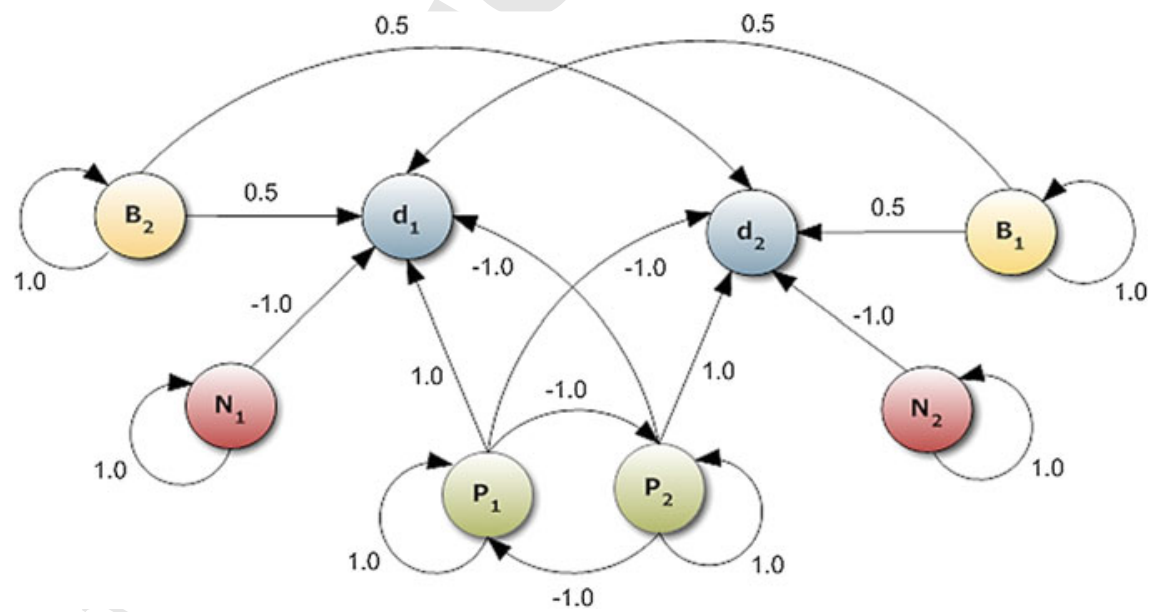

Fig. 1 The proposed Rough Cognitive Network for intrusion detection. The $d_{1}$ concept corresponds to the normal traffic class and the $d_{2}$ concept represents the abnormal traffic class. The $P_{i}$, $B_{i}$ and $N_{i}$ nodes denote the positive, boundary and negative regions for these two classes, $i \in\{1,2\}$ 
instance is classified as either "normal" or "abnormal"). More exactly, $d_{1}=$ "normal", $d_{2}=$ "abnormal", $P_{i}$ denotes the positive region associated to the $i$ th class, $N_{i}$ is the negative region related to the $i$ th class while $B_{i}$ is the $i$ th boundary region. Note that boundary concepts are allowed regardless of the inconsistency of the features in the target problem because only two decision classes are possible. More explicitly, if the problem has inconsistent instances, then both classes will be equally affected; otherwise, the activation value of the (empty) boundary regions will remain inactive during the inference process.

\subsubsection{Comparison with Traditional Classifiers Over KDDTest+}

The first experiment consists of studying the prediction ability of our model regarding the following set of traditional classifiers: J48 decision tree [54], NBTree [33], Random Forest [9], Random Tree [3], Multilayer Perceptron [56], Naive Bayes [28], and Support Vector Machine [12]. For experimental purposes, we adopted the first $20 \%$ of the records in KDDTrain+ for training all models. Figure 2a summarizes the accuracy achieved for each learner, whereas Fig. $2 \mathrm{~b}$ displays some representative samples of the solution space associated with the similarity threshold to be explored by the learning algorithm. In other words, Fig. $2 \mathrm{~b}$ illustrates the performance of our granular network for different similarity thresholds.

From the above experiment we can conclude that RCN results are competitive regarding J48 decision tree, Random Forest (RF), NBTree (NBT) and Random Tree (RT). However, our model outperforms other approaches such as Multilayer Perceptron (MLP), Naive Bayes (NB) and Support Vector Machine (SVM).

Next we study other statistics such as those extracted from the confusion matrices. True Negatives (TN) as well as True Positives (TP) correspond to correctly classified instances, that is, events that are rightly labeled as normal and attacks, respectively. Alternatively, False Positives (FP) refer to normal events being labeled as attacks while False Negatives (FN) are attack events incorrectly predicted as normal events. Table 1 shows such statistics for all classifiers used for comparison across the selected KDDTest+ dataset.

(a)

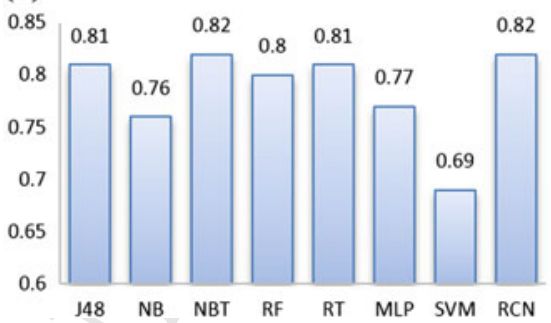

(b)

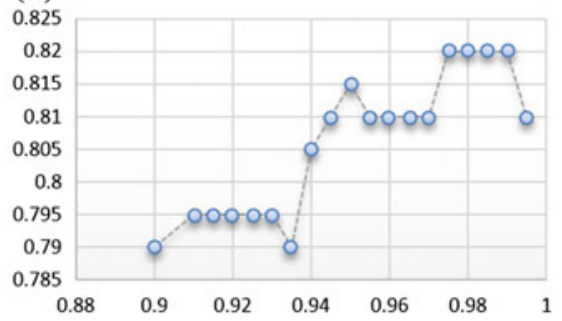

Fig. 2 Experiments using datasets KDDTrain+ and KDDTest+. a Accuracy of selected classifiers and $\mathbf{b}$ RCN accuracy as a function of the threshold values in Eq. (1) 
Table 1 Confusion matrix associated with each classifier for the KDDTest+ dataset

\begin{tabular}{l|l|l|l|l|l|l}
\hline & TN & FP & FN & TP & Detection rate & $\begin{array}{l}\text { False alarm } \\
\text { rate }\end{array}$ \\
\hline J48 & 9436 & 275 & 3996 & 8837 & 0.68 & 0.02 \\
\hline NB & 9010 & 701 & 4582 & 8251 & 0.64 & 0.07 \\
\hline NBT & 8869 & 842 & 3257 & 9576 & 0.74 & 0.08 \\
\hline RF & 9452 & 259 & 4523 & 8310 & 0.64 & 0.02 \\
\hline RT & 8898 & 813 & 3011 & 9822 & 0.76 & 0.08 \\
\hline MLP & 8971 & 740 & 4796 & 8037 & 0.62 & 0.07 \\
\hline SVM & 8984 & 727 & 4893 & 7940 & 0.61 & 0.07 \\
\hline RCN & 8891 & 820 & 3150 & 9683 & 0.75 & 0.08 \\
\hline
\end{tabular}

The reader may notice that $\mathrm{RCN}$ ranks as the second-best algorithm regarding the number of FN patterns. In our study we are especially interested in this value since it denotes the number of abnormal patterns that the IDS was unable to detect, although most authors prefer systems with high detection rate (i.e., $T P /(T P+F N))$ and low false alarm rate which is defined as $F P /(T N+F P)$. Nevertheless, in computer networks where high security is required, reducing the false negative rate is indispensable since only those patterns having normal features will be confidently allowed.

\subsubsection{Comparison with Traditional Classifiers Over KDDTest-21}

The second experiment is concerned with investigating the performance of our RCN model with respect to traditional classifiers, but now using the test set called KDDTest-21. Figure 3a portrays the classification accuracy achieved for each model while Fig. 3b displays the performance of the proposed granular network for different similarity thresholds.

(a)

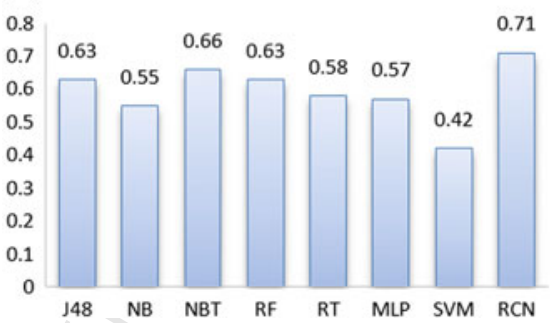

(b)

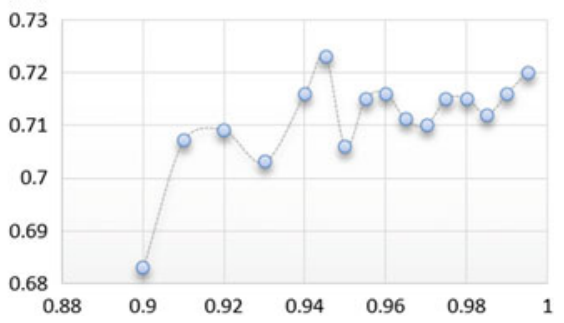

Fig. 3 Experiments using datasets KDDTrain+ and KDDTest-21. a Accuracy of selected classifiers and $\mathbf{b}$ RCN accuracy as a function of the threshold values in Eq. (1) 
Table 2 Confusion matrix associated with each classifier for the KDDTest-21 dataset

\begin{tabular}{l|l|l|l|l|l|l}
\hline & TN & FP & FN & TP & Detection rate & $\begin{array}{l}\text { False alarm } \\
\text { rate }\end{array}$ \\
\hline J48 & 1879 & 273 & 3996 & 5702 & 0.58 & 0.12 \\
\hline NB & 1460 & 692 & 4549 & 5149 & 0.53 & 0.32 \\
\hline NBT & 1354 & 798 & 3257 & 6441 & 0.66 & 0.37 \\
\hline RF & 1895 & 257 & 4523 & 5175 & 0.53 & 0.11 \\
\hline RT & 1388 & 764 & 3008 & 6690 & 0.68 & 0.35 \\
\hline MLP & 1426 & 726 & 4796 & 4902 & 0.50 & 0.33 \\
\hline SVM & 1440 & 712 & 4893 & 4805 & 0.49 & 0.33 \\
\hline RCN & 1572 & 580 & 2824 & 6874 & 0.70 & 0.26 \\
\hline
\end{tabular}

It should be specified that the KDDTest-21 dataset is more complex since it involves patterns that cannot be correctly classified by all learners. Despite this fact, our model was able to compute the best accuracy (71\%), notably outperforming the remaining approaches. However, in a previous experiment the model only achieved an accuracy of $66 \%$ due to the uncertainty present in the features during the inference stage (i.e., the overall evidence suggests accepting both decisions). To overcome this situation, we used the similarity classes pertaining to the $K$-nearest neighbors $(K=3)$ of the test instance $O_{i}$. In short, we adopted the similarity classes of its neighbors instead of only using the set $R\left(O_{i}\right)$ related to the target pattern for activating each input neuron in the network.

Table 2 shows the confusion matrix achieved by each classifier across the KDDTest-21 test set. In this case, our model computed the highest detection rate $(T P /(T P+F N)=0.7)$ and lowest false negative rate $(F N /(T P+F N)=0.29)$ which is the desired behavior. It means that the RCN will detect abnormal traffic with high accuracy, thus reducing the risk of classifying abnormal patterns as normal. In a nutshell, such statistics confirm the reliability of our granular classifier (RCN) for intrusion detection in complex computer networks. For instance, the reader may observe that if the false alarm rate is high, then the system will classify normal patterns as abnormal, but this behavior is preferable in order to avoid potential attacks.

\subsubsection{Discussion}

Although the above experiments show that RCNs are a suitable approach for addressing intrusion detection problems, there are cases where the inference suggests accepting a wrong decision class. This behavior could be a direct result of the strategy adopted for activating the input concepts, so other ways for estimating the activation vector could be explored. For example, in Bayesian inference one usually translates $\operatorname{Pr}(C \mid[x])$ into $\operatorname{Pr}(([x] C) \operatorname{Pr}(C)) / \operatorname{Pr}([x])$ by the Bayes theorem, which allows a practical estimation of initial conditions required to trigger the FCM inference process. 
Another aspect to be considered is related to the network weights, since rules $R_{1}-R_{5}$ formalize the direction (negative or positive) of each causal connection rather than its intensity. This means that the granular neural network discussed in this chapter calculates the decision class based on the initial state $A^{0}$ and the sign of causal relations, without exploiting the causal intensity. To achieve further performance gains, we are currently focused on computing this indicator via a supervised learning approach.

\section{Conclusions}

An important aspect in computer networks is how to detect intrusion since traditional approaches such as access control lists or firewalls are incapable of entirely protecting networks. In order to deal with such problem, several intrusion detection systems have been proposed; however, increasing the overall performance (e.g., the detection accuracy) is still an open problem for researchers. More explicitly, an essential component of intrusion detection systems is the inference algorithm used to classify network traffic patterns as either normal or abnormal. This problem could be thought of as a challenging binary classification task since modern intrusion techniques are sophisticated, so it is difficult to design models being able to distinguish between normal and abnormal patterns. As an example, frequently hackers attempt simulating trusted users in computer networks in order to gain access to remote resources. Such behavior will produce inconsistency in the collected traffic data; that is, objects that are very similar yet have been labeled as pertaining to different decision classes.

In this chapter we introduced a novel IDS based on Rough Cognitive Networks, a recently proposed granular neural network for pattern classification. Without loss of generality, we can define RCN as a Sigmoid Fuzzy Cognitive Map where input neurons represent information granules whereas output concepts denote decision classes. It should be remarked that the granulation of information is achieved by using Rough Sets, since it allows handling uncertainty arising from inconsistency. Furthermore, with the goal of increasing the reliability of the RCN-based inference process, we discussed a supervised learning methodology for automatically computing accurate similarity relations by estimating the proper parameter vector.

In order to measure the performance of our model, we adopted an improved version of the NSL-KDD dataset. From numerical simulations it is possible to conclude that our granular neural network is a suitable approach for detecting abnormal traffic patterns in computer networks. More precisely, we observed that RCNs are competitive regarding traditional classifiers such as $\mathrm{J} 48$ decision tree and Random Forest, across the simpler dataset (KDDTrain+). However, for the dataset KDDTest-21 the model significantly outperformed the other learners by computing the highest detection rate $(D R=0.7)$ and lowest false negative rate $(F N R=0.29)$. This confirms the reliability of the learning methodology put forth in this chapter to boost the model's performance. Future work along this front will concentrate on validating our approach on real computer networks. 


\section{References}

1. Abraham, A., Falcon, R., Bello, R.: Rough Set Theory: A True Landmark in Data Analysis. Springer, Heidelberg (2009)

2. Adetunmbi, A.O., Falaki, S.O., Adewale, O.S., Alese, B.K.: Network intrusion detection based on rough set and k-nearest neighbour. I. J. Comput. ICT Res. 2(1), 60-66 (2008)

3. Aldous, D.: The continuum random tree. I. Ann. Prob. 1-28 (1991)

4. Atzori, L., Iera, A., Morabito, G.: The internet of things: a survey. Comput. Netw. 54(15), 2787-2805 (2010)

5. Balajinath, B., Raghavan, S.: Intrusion detection through learning behavior model. Comput. Commun. 24(12), 1202-1212 (2001)

6. Bello, R., Falcon, R., Pedrycz, W., Kacprzyk, J.: Granular Computing: At The Junction of Rough Sets and Fuzzy Sets. Springer, Heidelberg (2008)

7. Bello, R., Verdegay, J.L.: Rough sets in the soft computing environment. Inf. Sci. 212, 1-14 (2012)

8. Bhuyan, M.H., Bhattacharyya, D., Kalita, J.K.: Network anomaly detection: methods, systems and tools. IEEE Commun. Surv. Tutorials 16(1), 303-336 (2014)

9. Breiman, L.: Random forests. Mach. Learn. 45(1), 5-32 (2001)

10. Bueno, S., Salmeron, J.L.: Benchmarking main activation functions in fuzzy cognitive maps. Expert Syst. Appl. 36(3), 5221-5229 (2009)

11. Cannady, J.: Artificial neural networks for misuse detection. In: National Information Systems Security Conference, pp. 368-81 (1998)

12. Chang, C.C., Lin, C.J.: Libsvm: a library for support vector machines. ACM Trans. Intell. Syst. Technol. (TIST) 2(3), 27 (2011)

13. Chen, R.C., Cheng, K.F., Chen, Y.H., Hsieh, C.F.: Using rough set and support vector machine for network intrusion detection system. In: First Asian Conference on Intelligent Information and Database Systems, 2009. ACIIDS 2009, pp. 465-470. IEEE (2009)

14. Chimphlee, W., Abdullah, A.H., Noor Md Sap, M., Srinoy, S., Chimphlee, S.: Anomaly-based intrusion detection using fuzzy rough clustering. In: International Conference on Hybrid Information Technology, 2006. ICHIT'06, vol. 1, pp. 329-334. IEEE (2006)

15. Costa, K.A., Pereira, L.A., Nakamura, R.Y., Pereira, C.R., Papa, J.P., Falcão, A.X.: A natureinspired approach to speed up optimum-path forest clustering and its application to intrusion detection in computer networks. Inf. Sci. 294, 95-108 (2015)

16. Dickerson, J.E., Dickerson, J.A.: Fuzzy network profiling for intrusion detection. In: 19th International Conference of the North American Fuzzy Information Processing Society, 2000. NAFIPS, pp. 301-306. IEEE (2000)

17. Elkan, C.: Results of the KDD'99 classifier learning. ACM SIGKDD Explor. Newsl. 1(2), 63-64 (2000)

18. Faraoun, K., Boukelif, A.: Genetic programming approach for multi-category pattern classification applied to network intrusions detection. Int. J. Comput. Intell. Appl. 6(01), 77-99 (2006)

19. Feng, W., Zhang, Q., Hu, G., Huang, J.X.: Mining network data for intrusion detection through combining svms with ant colony networks. Future Gener. Comput. Syst. 37, 127-140 (2014)

20. Gao, H.H., Yang, H.H., Wang, X.Y.: Ant colony optimization based network intrusion feature selection and detection. In: Proceedings of 2005 International Conference on Machine Learning and Cybernetics, 2005, vol. 6, pp. 3871-3875. IEEE (2005)

21. Geramiraz, F., Memaripour, A.S., Abbaspour, M.: Adaptive anomaly-based intrusion detection system using fuzzy controller. Int. J. Netw. Secur. 14(6), 352-361 (2012)

22. Govindarajan, M.: Hybrid intrusion detection using ensemble of classification methods. Int. J. Comput. Netw. Inf. Secur. 2, 45-53 (2014)

23. Guo, C., Zhou, Y., Ping, Y., Zhang, Z., Liu, G., Yang, Y.: A distance sum-based hybrid method for intrusion detection. Appl. Intell. 40(1), 178-188 (2014)

24. Hofmann, A., Schmitz, C., Sick, B.: Rule extraction from neural networks for intrusion detection in computer networks. In: IEEE International Conference on Systems, Man and Cybernetics, 2003, vol. 2, pp. 1259-1265. IEEE (2003) 
25. Hong, J., Baker, M.: Wearable computing. IEEE Pervasive Comput. 13(2), 7-9 (2014)

26. Jankowski, A., Skowron, A.: Toward perception based computing: A rough-granular perspective. In: Zhong, N., Liu, J., Yao, Y., Wu, J., Lu, S., Li, K. (eds.) Web Intelligence Meets Brain Informatics. Lecture Notes in Computer Science, vol. 4845, pp. 122-142. Springer, Heidelberg (2007)

27. Jazzar, M., Bin Jantan, A.: Using fuzzy cognitive maps to reduce false alerts in SOM-based intrusion detection sensors. In: Second Asia International Conference on Modeling Simulation, 2008. AICMS 08, pp. 1054-1060 (2008)

28. John, G.H., Langley, P.: Estimating continuous distributions in Bayesian classifiers. In: Proceedings of the Eleventh conference on Uncertainty in artificial intelligence, pp. 338-345. Morgan Kaufmann Publishers Inc. (1995)

29. Karami, A., Guerrero-Zapata, M.: A fuzzy anomaly detection system based on hybrid psokmeans algorithm in content-centric networks. Neurocomputing 149, 1253-1269 (2015)

30. KDD Cup 1999: KDD'99 dataset (2007). http://kdd.ics.uci.edu/databases/kddcup99/ kddcup99.html

31. Khan, M.S.A.: Rule based network intrusion detection using genetic algorithm. Int. J. Comput. Appl. 18(8), 26-29 (2011)

32. Kirkpatrick, K.: Software-defined networking. Commun. ACM 56(9), 16-19 (2013)

33. Kohavi, R.: Scaling up the accuracy of Naive-Bayes classifiers: a decision-tree hybrid. In: KDD, pp. 202-207 (1996)

34. Kosko, B.: Fuzzy cognitive maps. Int. J. Man Mach. Stud. 24(1), 65-75 (1986)

35. Kosko, B.: Hidden patterns in combined and adaptive knowledge networks. Int. J. Approximate Reasoning 2(4), 377-393 (1988)

36. Kosko, B.: Fuzzy Engineering (1996)

37. Krichene, J., Boudriga, N.: Incident response probabilistic cognitive maps. In: International Symposium on Parallel and Distributed Processing with Applications, 2008. ISPA '08, pp. 689-694 (2008)

38. Kuang, F., Xu, W., Zhang, S.: A novel hybrid KPCA and SVM with GA model for intrusion detection. Appl. Soft Comput. 18, 178-184 (2014)

39. Kuehn, A.: Extending Cybersecurity, Securing Private Internet Infrastructure: the US Einstein Program and its Implications for Internet Governance. Springer (2014)

40. Labib, K., Vemuri, V.R.: NSOM: A tool to detect denial of service attacks using self-organizing maps. Department of Applied Science University of California, Davis, California, USA, Technical Report (2002)

41. Li, L., Zhao, K.: A new intrusion detection system based on rough set theory and fuzzy support vector machine. In: 2011 3rd International Workshop on Intelligent Systems and Applications (ISA), pp. 1-5 (2011)

42. Liang, D., Pedrycz, W., Liu, D., Hu, P.: Three-way decisions based on decision-theoretic rough sets under linguistic assessment with the aid of group decision making. Appl. Soft Comput. 29, 256-269 (2015)

43. Liu, G.G.: Intrusion detection systems. In: Applied Mechanics and Materials, vol. 596, pp. 852-855. Trans Tech Publications (2014)

44. Loganathan, G.: A new heuristic optimization algorithm: harmony search. Simulation 76(2), 60-68 (2001)

45. Manikopoulos, C., Papavassiliou, S.: Network intrusion and fault detection: a statistical anomaly approach. IEE Commun. Mag. 40(10), 76-82 (2002)

46. McHugh, J.: Testing intrusion detection systems: a critique of the 1998 and 1999 DARPA intrusion detection system evaluations as performed by Lincoln laboratory. ACM Trans. Inf. Syst. Secur. 3(4), 262-294 (2000)

47. Mell, P., Grance, T.: The NIST definition of cloud computing (2011)

48. Nápoles, G., Grau, I., Vanhoof, K., Bello, R.: Hybrid model based on rough sets theory and fuzzy cognitive maps for decision-making. In: Kryszkiewicz, M., Cornelis, C., Ciucci, D., Medina-Moreno, J., Motoda, H., Ras, Z. (eds.) RSEISP 2014 (2014)

49. Pawlak, Z.: Rough sets. Int. J. Comput. Inf. Sci. 11(5), 341-356 (1982) 
50. Pedrycz, W., Al-Hmouz, R., Morfeq, A., Balamash, A.S.: Building granular fuzzy decision support systems. Knowl.-Based Syst. 58, 3-10 (2014)

51. Pedrycz, W., Al-Hmouz, R., Morfeq, A., Balamash, A.S.: Distributed proximity-based granular clustering: towards a development of global structural relationships in data. Soft Comput. 1-17 (2014)

52. Pedrycz, W., Skowron, A., Kreinovich, V.: Handbook of Granular Computing. Wiley (2008)

53. Poongothai, T., Duraiswamy, K.: Effective cross layer intrusion detection in mobile ad hoc networks using rough set theory and support vector machines. Asian J. Inf. Technol. 12(8), 242-249 (2013)

54. Quinlan, J.R.: C4.5: Programs for Machine Learning (2014)

55. Roh, S.B., Pedrycz, W., Ahn, T.C.: A design of granular fuzzy classifier. Expert Syst. Appl. 41(15), 6786-6795 (2014)

56. Ruck, D.W., Rogers, S.K., Kabrisky, M., Oxley, M.E., Suter, B.W.: The multilayer perceptron as an approximation to a Bayes optimal discriminant function. IEEE Trans. Neural Netw. 1(4), 296-298 (1990)

57. Shafi, K., Abbass, H.A.: Biologically-inspired complex adaptive systems approaches to network intrusion detection. Inf. Secur. Tech. Rep. 12(4), 209-217 (2007)

58. Shafi, K., Abbass, H.A.: An adaptive genetic-based signature learning system for intrusion detection. Expert Syst. Appl. 36(10), 12036-12043 (2009)

59. Shafi, K., Kovacs, T., Abbass, H.A., Zhu, W.: Intrusion detection with evolutionary learning classifier systems. Nat. Comput. 8(1), 3-27 (2009)

60. Shrivastava, S.K., Jain, P.: Effective anomaly based intrusion detection using rough set theory and support vector machine. Int. J. Comput. Appl. 18(3), 35-41 (2011)

61. Simmross-Wattenberg, F., Asensio-Pérez, J.I., Casaseca-de-la H.P., Martin-Fernandez, M., Dimitriadis, I.A., Alberola-Lopez, C.: Anomaly detection in network traffic based on statistical inference and alpha-stable modeling. IEEE Trans. Dependable Secure Comput. 8(4), 494-509 (2011)

62. Siraj, A., Vaughn, R.: Multi-level alert clustering for intrusion detection sensor data. In: Annual Meeting of the North American Fuzzy Information Processing Society, 2005. NAFIPS 2005, pp. 748-753 (2005)

63. Siraj, A., Bridges, S.M., Vaughn, R.B.: Fuzzy cognitive maps for decision support in an intelligent intrusion detection system. In: Joint 9th IFSA World Congress and 20th NAFIPS International Conference, 2001, vol. 4, pp. 2165-2170. IEEE (2001)

64. Siraj, A., Vaughn, R.B., Bridges, S.M.: Intrusion sensor data fusion in an intelligent intrusion detection system architecture. In: Proceedings of the 37th Annual Hawaii International Conference on System Sciences, 2004, pp. 1-10. IEEE (2004)

65. Sivaranjanadevi, P., Geetanjali, M., Balaganesh, S., Poongothai, T.: An effective intrusion system for mobile ad hoc networks using rough set theory and support vector machine. IJCA Proc. E Governance Cloud Comput. Serv. 2, 1-7 (2012)

66. Song, X., Wu, M., Jermaine, C., Ranka, S.: Conditional anomaly detection. IEEE Trans. Knowl. Data Eng. 19(5), 631-645 (2007)

67. Sun, J., Yang, H., Tian, J., Wu, F.: Intrusion detection method based on wavelet neural network. In: Second International Workshop on Knowledge Discovery and Data Mining, 2009. WKDD 2009, pp. 851-854. IEEE (2009)

68. Tajbakhsh, A., Rahmati, M., Mirzaei, A.: Intrusion detection using fuzzy association rules. Appl. Soft Comput. 9(2), 462-469 (2009)

69. Tavallaee, M., Bagheri, E., Lu, W., Ghorbani, A.A.: A detailed analysis of the KDD CUP 99 data set. In: Proceedings of the Second IEEE Symposium on Computational Intelligence for Security and Defence Applications 2009 (2009)

70. Visconti, A., Tahayori, H.: Artificial immune system based on interval type-2 fuzzy set paradigm. Appl. Soft Comput. 11(6), 4055-4063 (2011)

71. Wang, C.M., Huang, Y.F.: Self-adaptive harmony search algorithm for optimization. Expert Syst. Appl. 37(4), 2826-2837 (2010) 
72. Wang, W., Pedrycz, W., Liu, X.: Time series long-term forecasting model based on information granules and fuzzy clustering. Eng. Appl. Artif. Intell. 41, 17-24 (2015)

73. Wu, S.X., Banzhaf, W.: The use of computational intelligence in intrusion detection systems: a review. Appl. Soft Comput. 10(1), 1-35 (2010)

74. Xin, J., Dickerson, J., Dickerson, J.A.: Fuzzy feature extraction and visualization for intrusion detection. In: The 12th IEEE International Conference on Fuzzy Systems, 2003. FUZZ'03, vol. 2, pp. 1249-1254. IEEE (2003)

75. Yang, H., Li, T., Hu, X., Wang, F., Zou, Y.: A survey of artificial immune system based intrusion detection. Sci. World J. 2014 (2014)

76. Yao, Y.: Three-way decision: An interpretation of rules in rough set theory. Lecture Notes in Computer Science (including subseries Lecture Notes in Artificial Intelligence and Lecture Notes in Bioinformatics) 5589 LNAI, 642-649 (2009)

77. Yao, Y.: Three-way decisions with probabilistic rough sets. Inf. Sci. 180(3), 341-353 (2010)

78. Yong, H., Feng, Z.X.: Expert system based intrusion detection system. In: 2010 International Conference on Information Management, Innovation Management and Industrial Engineering (ICIII), vol. 4, pp. 404-407. IEEE (2010)

79. Yu, M.: A nonparametric adaptive cusum method and its application in network anomaly detection. Int. J. Advancements Comput. Technol. 4(1), 280-288 (2012)

80. Zaghdoud, M., Al-Kahtani, M.S.: Contextual fuzzy cognitive map for intrusion response system. Int. J. Comput. Inf. Technol. 2(3), 471-478 (2013)

81. Zhang, C., Jiang, J., Kamel, M.: Comparison of BPL and RBF network in intrusion detection system. In: Rough Sets, Fuzzy Sets, Data Mining, and Granular Computing, pp. 466-470. Springer (2003)

82. Zhang, L., Bai, Z., Luo, S., Cui, G., Li, X.: A dynamic artificial immune-based intrusion detection method using rough and fuzzy set. In: 2013 International Conference on Information and Network Security (ICINS 2013), pp. 1-7 (2013)

83. Zhong, C., Yang, F., Zhang, L., Li, Z.: An efficient distributed coordinated intrusion detection algorithm. In: 2005 International Conference on Machine Learning and Cybernetics, pp. 26792685 (2006) 